\title{
Patients Undergoing Ileoanal Pouch Surgery Experience a Constellation of Symptoms and Consequences Representing a Unique Syndrome
}

\author{
A Report From the Patient-Reported Outcomes After Pouch Surgery \\ (PROPS) Delphi Consensus Study
}

Paul Cavallaro, MD, ${ }^{*}$ Nicola Fearnhead, DM, $\dagger$ Ian Bissett, MD, $\ddagger$ Mantaj Brar, MD, $\S$ Thomas Cataldo, MD, $\uparrow$ Rasheed Clarke,|| Paula Denoya, MD, ${ }^{* *}$ Amber Lorraine Elder, †† Krisztina Gecse, MD, Samantha Hendren, MD, MPH, §§ Stefan Holubar, MD, $\uparrow$ Nimalan Jeganathan, MD,|||| Pär Myrelid, MD, ${ }^{* * *}$

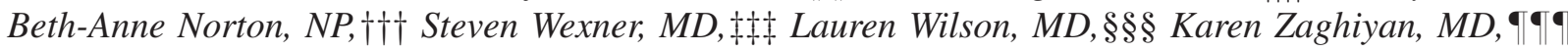
and Liliana Bordeianou, MD, MPH||||||凶, On behalf of the PROPS Delphi Study Expert Panels**

From the *Department of General Surgery, Massachusetts General Hospital, Boston, Massachusetts; $\nmid$ Department of Colorectal Surgery, Cambridge University Hospitals NHS Foundation Trust, Cambridge, UK; $\ddagger$ Department of Surgery, University of Auckland, Auckland, New Zealand; §Division of General Surgery, Department of Surgery, Mount Sinai Hospital, University of Toronto, Toronto, Canada; - Division of Colon \& Rectum Surgery, Beth Israel-Deaconess Health Medical Center, Harvard Medical School, Boston, Massachusetts; ||Patient Advocate, Blogger, www.rasheedclarke.com; **Division of Colon and Rectal Surgery, Department of Surgery, Stony Brook University Hospital, Stony Brook, New York; ††Patient Advocate, Blogger, www.colitisninja.com; $\ddagger \ddagger D e p a r t m e n t ~ o f$ Gastroenterology and Hepatology, Amsterdam UMC, University of Amsterdam, Meibergdreef 9, Amsterdam, the Netherlands; §§Department of Surgery, University of Michigan, Ann Arbor, Michigan; ' 'Department of Colon and Rectal Surgery, Cleveland Clinic, Cleveland, Ohio; ||||Department of Surgery, Division of Colon \& Rectal Surgery, Pennsylvania State University College of Medicine, Hershey, Pennsylvania; ${ }^{* * *}$ Department of Surgery, County Council of Östergötland, and Department of Biomedical and Clinical Sciences, Linköping University, Linköping, Sweden; ††Crohn's and Colitis Center, Brigham and Women's

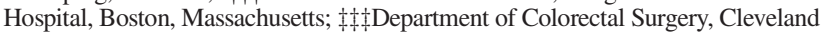
Clinic Florida, Weston, Florida; §§§Department of Surgery, Dartmouth-Hitchcock Medical Center, Lebanon, New Hampshire; ब बDivision of Colorectal Surgery, Cedars-Sinai Medical Center, Los Angeles, California; and ||||||MGH Colorectal Surgery and Crohn's and Colitis Centers, Department of Gastrointestinal Surgery and Surgical Oncology, Boston, Massachusetts.

\lbordeianou@mgh.harvard.edu.

**Appendix A, http://links.lww.com/SLA/C990.

Paul Cavallaro and Nicola Fearnhead are co-first authors.

This study was funded by a grant from the Crohn's and Colitis Foundation Surgical Research Network. Additional logistical support was provided by the Tripartite 2020 Vision Group, which is supported by the American Society of Colorectal Surgery, the Association of Coloproctology of Great Britain and Ireland, the Colorectal Surgical Society of Australia and New Zealand and the European Society of Coloproctology to encourage international, patient-centered, colorectal surgery research.

This article is being published concurrently in Annals of Surgery \& Diseases of the Colon and Rectum. The articles are identical except for minor stylistic and spelling differences in keeping with each journal's style. Citation from any of the 2 journals can be used when citing this article.

Podium presentation at the annual meeting of the The American Society of Colon and Rectal Surgeons [Virtual Meeting], April 24-28, 2021.

WTe authors report no conflicts of interest.

్․․ Author Contributions: Paul Cavallaro, MD: (1) conception and design, acquisition of data, analysis and interpretation of data; (2) drafting of manuscript and revising it critically for important intellectual content; and (3) final approval of the version to be published.

Nicola Fearnhead, DM: (1) conception and design, analysis and interpretation of data; (2) drafting of manuscript and revising it critically for important intellectual content; and (3) final approval of the version to be published.

Ian Bissett, MD: (1) analysis and interpretation of data; (2) Critical revising of manuscript for important intellectual content; and (3) final approval of the version to be published.

Mantaj Brar, MD: (1) analysis and interpretation of data; (2) Critical revising of manuscript for important intellectual content; and (3) final approval of the version to be published.
Thomas Cataldo, MD: (1) analysis and interpretation of data; (2) Critical revising of manuscript for important intellectual content; and (3) final approval of the version to be published.

Rasheed Clarke: (1) analysis and interpretation of data; (2) Critical revising of manuscript for important intellectual content; and (3) final approval of the version to be published.

Paula Denoya, MD: (1) analysis and interpretation of data; (2) Critical revising of manuscript for important intellectual content; and (3) final approval of the version to be published.

Amber Lorraine Elder: (1) analysis and interpretation of data; (2) Critical revising of manuscript for important intellectual content; and (3) final approval of the version to be published.

Krisztina Gecse, MD: (1) analysis and interpretation of data; (2) Critical revising of manuscript for important intellectual content; and (3) final approval of the version to be published.

Samantha Hendren, MD, MPH: (1) analysis and interpretation of data; (2) Critical revising of manuscript for important intellectual content; and (3) final approval of the version to be published.

Stefan Holubar, MD: (1) analysis and interpretation of data; (2) Critical revising of manuscript for important intellectual content; and (3) final approval of the version to be published.

Nimalan Jeganathan, MD: (1) analysis and interpretation of data; (2) Critical revising of manuscript for important intellectual content; and (3) final approval of the version to be published.

Pär Myrelid, MD: (1) analysis and interpretation of data; (2) Critical revising of manuscript for important intellectual content; and (3) final approval of the version to be published.

Beth-Anne Norton, NP: (1) analysis and interpretation of data; (2) Critical revising of manuscript for important intellectual content; and (3) final approval of the version to be published.

Steven Wexner, MD: (1) analysis and interpretation of data; (2) Critical revising of manuscript for important intellectual content; and (3) final approval of the version to be published.

Lauren Wilson, MD: (1) analysis and interpretation of data; (2) Critical revising of manuscript for important intellectual content; and (3) final approval of the version to be published.

Karen Zaghiyan, MD: (1) analysis and interpretation of data; (2) Critical revising of manuscript for important intellectual content; and (3) final approval of the version to be published.

Liliana Bordeianou, MD: (1) conception and design, acquisition of data, analysis and interpretation of data; (2) drafting of manuscript and revising it critically for important intellectual content; and (3) final approval of the version to be published.

Supplemental digital content is available for this article. Direct URL citations appear in the printed text and are provided in the HTML and PDF versions of this article on the journal's Web site (www.annalsofsurgery.com).

This is an open access article distributed under the terms of the Creative Commons Attribution-Non Commercial-No Derivatives License 4.0 (CCBY-NC-ND), where it is permissible to download and share the work provided it is properly cited. The work cannot be changed in any way or used commercially without permission from the journal.

Copyright (C) 2021 The Author(s). Published by Wolters Kluwer Health, Inc.

ISSN: 0003-4932/21/27401-0138

DOI: $10.1097 /$ SLA.0000000000004829 
Objective: The primary aim was to create a patient-centered definition of core symptoms that should be included in future studies of pouch function. Background: Functional outcomes after ileoanal pouch creation have been studied; however, there is great variability in how relevant outcomes are defined and reported. More importantly, the perspective of patients has not been represented in deciding which outcomes should be the focus of research.

Methods: Expert stakeholders were chosen to correlate with the clinical scenario of the multidisciplinary team that cares for pouch patients: patients, colorectal surgeons, gastroenterologists/other clinicians. Three rounds of surveys were employed to select high-priority items. Survey voting was followed by a series of online patient consultation meetings used to clarify voting trends. A final online consensus meeting with representation from all 3 expert panels was held to finalize a consensus statement.

Results: One hundred ninety-five patients, 62 colorectal surgeons, and 48 gastroenterologists/nurse specialists completed all 3 Delphi rounds. Fiftythree patients participated in online focus groups. One hundred sixty-one stakeholders participated in the final consensus meeting. On conclusion of the consensus meeting, 7 bowel symptoms and 7 consequences of undergoing ileoanal pouch surgery were included in the final consensus statement.

Conclusions: This study is the first to identify key functional outcomes after pouch surgery with direct input from a large panel of ileoanal pouch patients. The inclusion of patients in all stages of the consensus process allowed for a true patient-centered approach in defining the core domains that should be focused on in future studies of pouch function.

Keywords: ileoanal pouch, inflammatory bowel disease, patient reported outcomes, ulcerative colitis

(Ann Surg 2021;274:138-145)

$\mathrm{R}$ storative proctocolectomy with ileal-pouch anal anastomosis IPAA) in a J-pouch configuration was first described more than 40 years ago. ${ }^{1}$ In the last 4 decades, it has become the most commonly performed operation for patients with ulcerative colitis (UC) requiring surgery. Long-term functional outcomes after ileoanal pouch creation have been studied, ${ }^{2-5}$ however the field lacks standardization in reporting of functional measures, with the literature being mostly focused on fecal incontinence (FI) and frequency of bowel movements. In contrast, many studies have been focused on patients with "low anterior resection syndrome" after proctectomy for rectal cancer, demonstrating additional bowel dysfunction symptoms and consequences. ${ }^{6,7}$ Because UC patients undergo both proctectomy and total colectomy, their bowel function after surgery is influenced both by loss of colonic water absorption and by loss of rectal reservoir functions. Thus this complex postoperative setting needs to be comprehensively explored at functional level. ${ }^{8}$

Unfortunately, patients have frequently been absent from discussions concerning which outcomes should be studied after colorectal surgery. In an enlightening study, Brandsborg et $\mathrm{al}^{9}$ evaluated differences in perception of pouch dysfunction between clinicians (31 surgeons and 12 gastroenterologists) and patients with striking results - clinicians performed no better than random probability at choosing the 5 most important symptoms to patients out of a list of 12 symptoms generated by patients. Clinicians tended to overestimate the importance of frequent bowel movements and seepage of stool, 2 of the most widely reported ileoanal pouch surgery outcomes, while underestimating the importance of urgency and incomplete evacuation. Although patients historically have had little stake in studies of functional outcomes, the importance of their perceptions of their own function, particularly when being researched, should be paramount. ${ }^{10}$

With these considerations in mind, the aim of this study was to determine which symptoms both patients and experts consider important for evaluating pouch function. We used established Delphi consensus methodology and ensured patients as the key stakeholders in the process. We thus sought to create a patient-centered core outcome set that could then be utilized in reporting all future studies of pouch function.

\section{METHODS}

\section{Scientific Committee}

Nine members of the Crohn's and Colitis Foundation (CCF) Surgical Research Network were designated as the initial Scientific Committee. Additional representation from Canada, UK, Europe, and Australasia was then obtained by invitation based on reputation as international experts in the field. These clinicians also helped identify and recruit nurse specialists, patient advocates, and gastroenterologists to serve on the scientific committee. Ethical approval for the study was granted by the Massachusetts General Hospital Internal Review Board (Protocol: 2019P000671). All patient registration and data capture were completed using the REDcap electronic platform. $^{11}$

\section{Expert Panels}

Three "expert" panels in considering ileoanal pouch surgery were recruited: patients with IPAAs, surgeons, and gastroenterologists/other specialists (clinicians) who normally care for IPAA patients before and after surgery. In the interest of maintaining a patient-centered process, an a priori decision was made to aim for a minimum 1:1 recruitment ratio of patients to providers, anticipating recruitment of 120 patients and no more than 120 clinicians. Patients, surgeons, and clinicians were recruited via the CCF clinician research networks and patient advocacy groups as detailed below. Maximum diversity sampling (nonprobabilistic purposive sampling) was used to recruit clinicians with a wide range of experience and perspectives (Appendix A, http://links.lww.com/SLA/C990).

Patients were eligible to participate if they were over the age of 18 and had undergone IPAA with any reconstruction (J, S, W-pouch) for any diagnosis before the study date (including UC, Crohn disease, and familial adenomatous polyposis). Patients had to have had intestinal continuity restored (by reversal of protective diverting ileostomy) for at least 1 year. Patients were excluded if they had pouch failure resulting in removal of their pouch more than 3 years before the study or were not able to complete the survey in English. Patients were recruited by Institutional Review Board-approved advertisements on the social media pages (Facebook, twitter) of the CCF and other inflammatory bowel disease (IBD) and/or UC patient advocacy groups. Patients volunteered for the study by registering online after reading an information page. Patient participants completed a registration form to obtain demographic details and baseline clinical information surrounding their diagnosis and IPAA to facilitate identification of inclusion/exclusion criteria.

Surgeons were recruited through the Crohn's and Colitis Surgery Research Network, and gastroenterologists were recruited via the Crohn's and Colitis Clinical Research Alliance (http:// www.ibdclinicalresearchnetworks.org/). These networks allow for a selection of clinicians with explicit interest and expertise in the care of patients with IBD.

\section{Delphi Domain Generation}

The scientific committee oversaw initial domain generation and subsequent arbitration of study related questions. An electronic search was performed in the Pubmed, Medline, EMBASE, and Cochrane Library databases up to June 1st, 2019, to identify all of the relevant articles using key terms separated by Boolean operators including ulcerative colitis, ileal pouch anal anstomosis, $\mathrm{J}$ pouch, functional outcomes, and bowel function. A manual search of the reference lists of included studies was performed to identify 


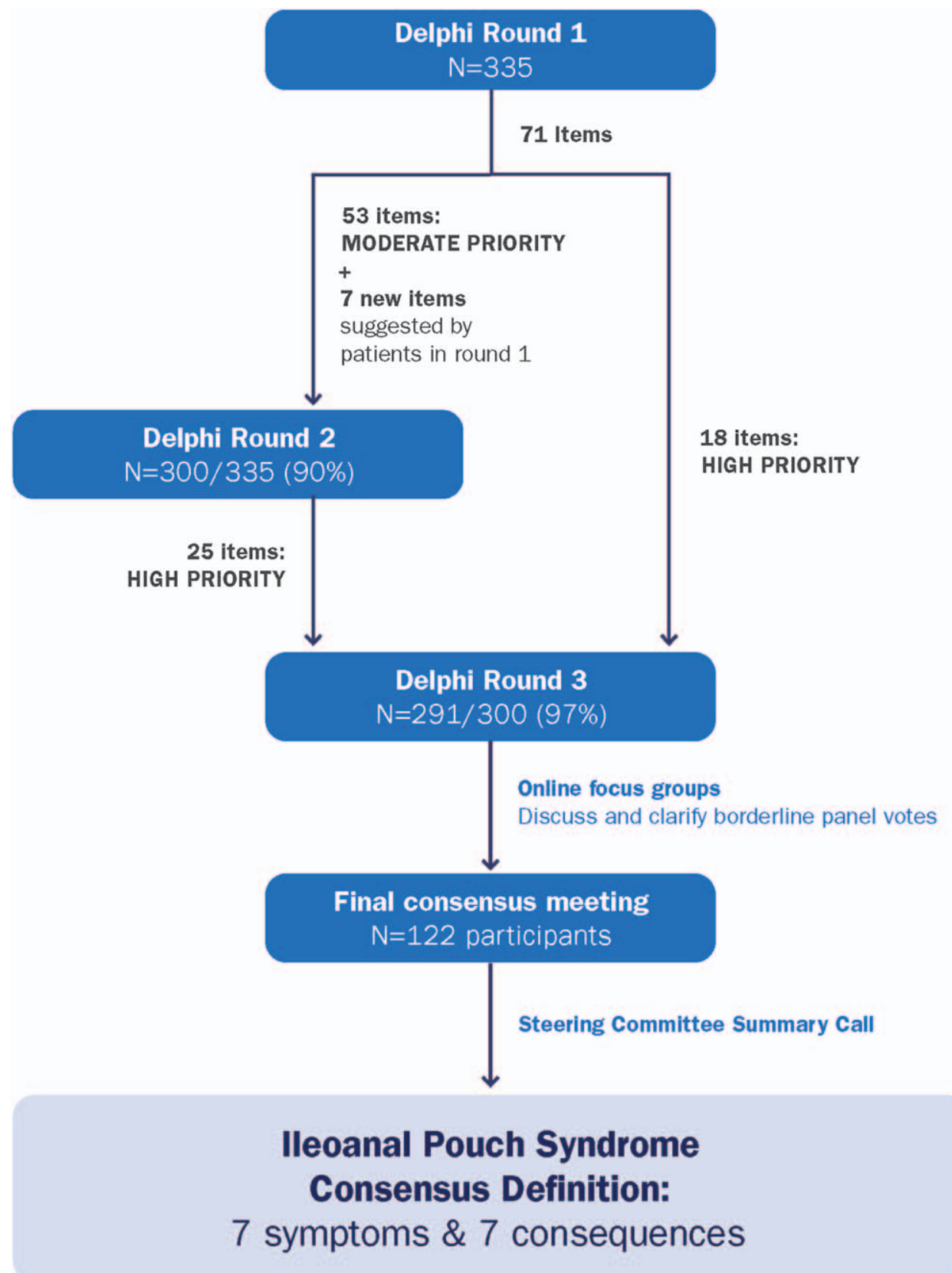

FIGURE 1. Study schema depicting progression of the consensus process through 3 Delphi rounds, patient focus groups, and a final consensus meeting.

additional relevant articles with functional outcomes or consequences. Additional domains were generated via interview with patients and patient advocates.

\section{Delphi Survey Administration}

Three rounds of online Delphi surveys were used to identify high-priority outcomes to include in the definition of IPS. A study schema is shown in Figure 1. Delphi methodology involves a structured iterative communication process that relies on a panel of "experts" to systematically reach a consensus decision. In each of the 3 rounds of voting, participants were shown a domain and asked, "In your opinion, how important is this factor for a patient's overall experience of having a pouch?" Participants scored each domain using a numerical Likert scale from 1 to 9 or "not applicable."
(Fig. 2). They were given the instruction that 9 is the most important (in other words, it has the greatest effect on patients) and 1 is the least important (if present, it would have less impact on patients). Rankings of 7-9 indicate items of high priority, ratings of 4-6 were of moderate priority ("important but not essential"), and rankings of $1-$ 3 were considered low priority. For the patient panel specifically, participants were instructed to score the importance of each factor regardless of how severe this symptom may be for them personally. For example, if a participant feels a factor is very important to overall function, but they do not necessarily experience that factor, they would still rank this symptom with a score of 7-9. Each round was open for 4 weeks and weekly reminder emails were sent to nonresponders. Each outcome was evaluated for heterogeneity using a histogram for each group of the number of scores that fell into the 

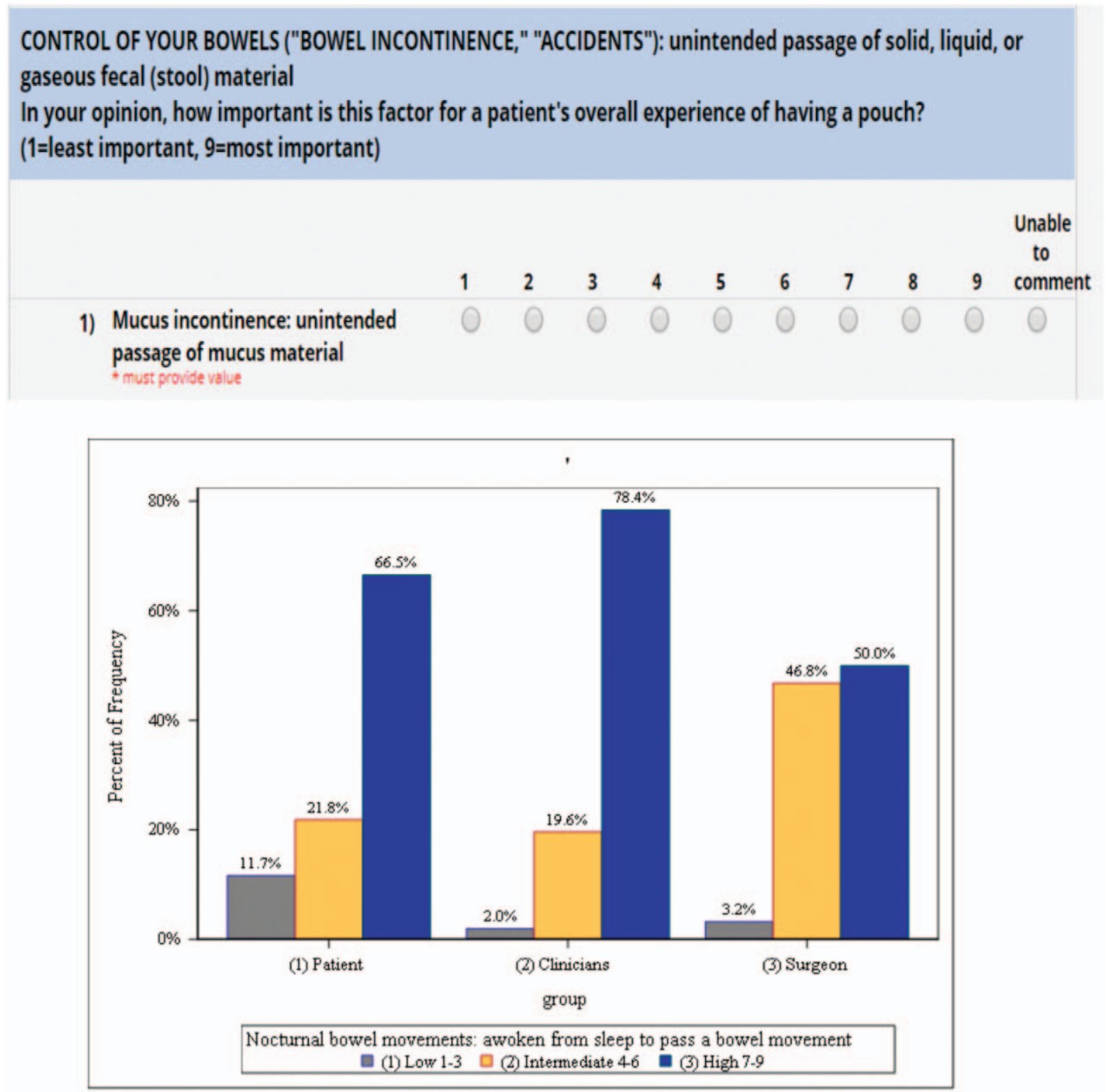

FIGURE 2. Delphi round 2 question example, with accompanying histogram to demonstrate responses by expert group.

high, moderate, or low priority categories. Participants were shown the distribution of each groups' scores from the previous round for each question in the subsequent round (Fig. 2).

Domains scored as low priority (Likert $1-3$ ) by a majority of participants were removed from the survey process after each round. Domains from the first round were progressed forward directly to the final third round if over $67 \%$ of all participants in the patient panel scored the item as high priority, or if there was concordance between the surgeon and GI/other clinician panel with $>67 \%$ scoring highpriority in both groups. Domains scoring in the moderate priority range in the first round were reviewed and then reworded, consolidated, or clarified as necessary, and then included in the second Delphi round. Domains scoring high-priority by over $67 \%$ of participants in the second round were also added to the third and final round. Thus, all "high priority" items identified in the first and second round were then voted on again in the final third round, where a final majority vote (50\% agreement) advanced the item for discussion to the final consensus meeting.

\section{Patient Focus Group Discussions}

Following 3 rounds of voting and before the final consensus meeting, the patient cohort was invited to participate in online small focus group discussions using the Zoom web-based platform and nominal group technique (Ref: https://www.cdc.gov/healthyyouth/ evaluation/pdf/brief7.pdf). Four separate virtual focus groups were convened, each with no more than 15 patient participants. These allowed for in-depth conversations and clarification of sensitive topics and concepts. Patients were asked structured questions and their answers were tabulated and summarized for discussion at the final consensus meeting to allow for further interpretation of the 
Delphi data. Discussion focused on items where some discrepancy was noted between expert panels.

\section{Final Consensus Meeting}

A final virtual consensus meeting was held using the Zoom web-based conference platform. All patients and clinicians who completed the 3 Delphi rounds were invited to participate and the conference results from the 3 Delphi rounds and the online patient focus group themes were presented and discussed. This was followed by a final vote on how to word or potentially amalgamate various concepts into domains.

\section{Data Analysis}

Variables are presented as median (interquartile range) or count (percentage) as appropriate. The Chi-square test was used to compare categorical data. All statistical analysis was performed using Stata software, version SE 14.0 (StataCorp, College Station, TX). All tests were 2 -sided and statistical significance was accepted at the $P<0.05$ level.

\section{RESULTS}

\section{Expert Panel Description}

The expert panels exceeded initial recruitment goals and included 217 patient volunteers, whereas the surgeon and clinician panel had 62 surgeons and 56 gastroenterologists/other clinician volunteers respectively (Fig. 3A and B). Overall, 87\% (291/335) of participants completed all 3 rounds of surveys. The majority of patients had J-pouches for UC and were satisfied with pouch function. All but $11.5 \%$ of patients would recommend a pouch to another patient. Surgeons were likely to be colorectal fellowship trained, have at least 5 years of experience, and perform a median of 5 pouch operations per year. Similarly, most gastroenterologists had over 5 years of experience and over $60 \%$ of their practice focused on IBD patients. A full list of clinician participants and affiliations is shown in Appendix A, http://links.lww.com/SLA/C990.

\section{Online Delphi Survey Results}

Round 1 included 71 questions. Respondents identified 18 items as "high priority," grouping into the general concepts of fecal incontinence, soiling, urgency, and perianal pain. Moderate priority items, and new items suggested by patients in the first round, were reviewed and then again voted on in the second round. This yielded an additional 25 "high priority" items grouping in the general concepts of nocturnal symptoms, social-emotional consequences, impact on intimacy, and impact on sleep/energy, among others. In the third and final round of surveys, participants were asked to pick the most important of the high-priority items by being as discriminatory as possible. In this round, the threshold of a majority $(>50 \%)$ vote from the patient panel was used to allow items to automatically be included in the definition of IPS. Items where other panels voted higher than $50 \%$ were also included for discussion. See Appendices B, http://links.lww.com/SLA/C991 and C, http://links.lww.com/ SLA/C992 for specific details of how each domain progressed through each round of voting. Appendix D, http://links.lww.com/ SLA/C993 displays a heat map with the proportion of panelists that voted each item as high-priority on round 3 , compared to results from rounds 1 and 2 .

\section{Patient Focus Groups}

Overall, 53 patients participated in 4 virtual focus groups. Several important themes emerged. For example, there was general agreement that multiple bowel movements are a universal aspect of life with a pouch and should be included in the final definition, even though patients did not consider the symptom bothersome unless their number was "excessive." The concept of accommodations to life with a pouch, including dietary and medication accommodations, was widely viewed as a critical component that may have been missed in how the survey question was posited. The group felt that these should be combined into 1 domain. Perianal pain was viewed by all participants as highly impactful and likely subject to vote splitting and confusion with other forms of pain included in survey items. Patterns of incomplete bowel movements (clustering or fragmentation) were identified by many patients as important aspects of function, but likely to have been subject to vote splitting. Lastly, many patients expressed concern that although their quality of life, ability to hold relationships, feelings on intimacy, etc actually improved after their pouch when compared to before surgery, many of these adjustments and compromises were still required and were important to include as core outcomes for future studies of pouch function.

\section{Final Consensus Meeting}

One hundred twenty-two participants $(73 \%$ patients, $18 \%$ surgeons, $9 \%$ gastroenterologists) participated in the final virtual consensus meeting. Discussion was structured around domains that had met consensus or were identified as important by the focus groups. Items that could be amalgamated or items that had discrepancy between groups were discussed in detail. Real-time polling using the Zoom platform was utilized to identify whether a consensus had been reached, defined as $70 \%$ of attendees voting for a motion. Visual aids in the form of a PowerPoint presentation with graphical representation of the data and patient participant quotes were included to ensure a patient-centered voice in the meeting. During discussion of each domain, patients were provided the initial opportunity to share opinions on each item.

\section{lleoanal Pouch Syndrome Definition}

The consensus meeting discussion was summarized by the scientific committee. Seven symptoms and 7 accommodations/consequences of an ileoanal pouch were included into the final consensus statement. Ultimately, the patient panel, in conjunction with the surgeon and gastroenterology stakeholders participating in the consensus meeting, coined the term "ileoanal pouch syndrome" (IPS), that is, the amalgamation of symptoms that describe the full range of expected function after creation of ileoanal pouch, while highlighting those symptoms that may have a negative impact on the quality of life of pouch patients and thus need recognition, education, and treatment. These symptoms are summarized in Figure 4. Full definitions of each symptom and accommodation, and examples of how these were described by patients in their own words, are provided in Appendix E, http://links.lww.com/SLA/C994. The actual impact (positive, negative, or neutral) on quality of life was not measured.

\section{Additional Core Outcomes}

Five additional items were identified as important but outside of the scope of the project and were recommended by participants as suggested topics for future research. These include: (1) Sexuality, sexual function, and dysfunction; risks and mitigation. (2) Conception/ pregnancy/delivery risks. (3) Pouchitis causes and treatment, and its differentiation from normal or abnormal pouch function. (4) Best practice in delivery of services and for long-term follow up after pouch surgery. (5) Proper consent before surgery, including counseling and education before and after pouch, lack of support group of pouch patients, and perception of being "forgotten" after being "cured."

\section{DISCUSSION}

Initiatives to generate patient-centered core outcome sets have recently increased in prevalence based on the principles outlined 
Patient Characteristics $\mathrm{N}=217$

Age (median, IQR): $39(31-49)$
Missing (4.6\%)

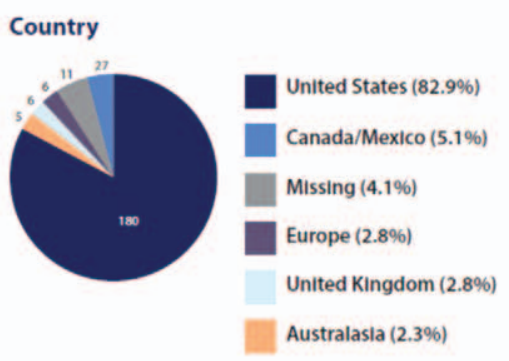

A

\section{Surgeons $\mathrm{N}=67$}

Board-certified Colorectal Surgeons: 48 (77.4\%)
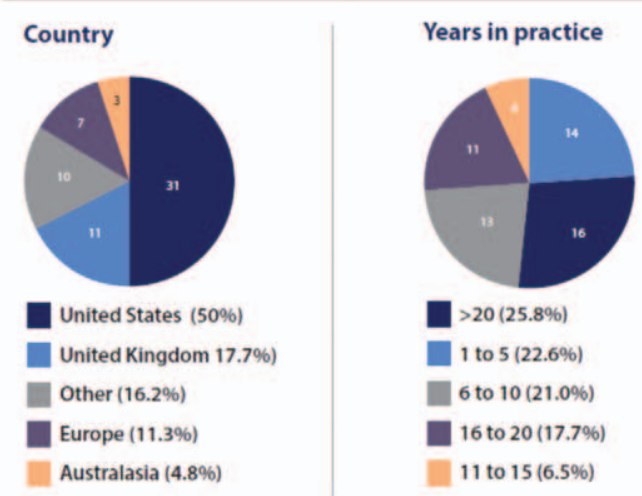

$\%$ of practice managing IBD patients: 30 (19-50\%)

Approx. J-pouches created per year: 5 (3-13)

Perceived patient satisfaction after pouch surgery

Poor: $0(096)$ 1 YEAR

Poor: 0 (0\%)

Fair: $5(8.1 \%)$ Good: $35(56.4 \%)$ Good: $26(41.9 \%)$ Very Good: $9(14.5 \%)$ Very Good: $19(30.6 \%)$ Excellent: $3(4.8 \%)$

3 YEARS
Poor: $0(0 \%)$
Fair: $7(11.3 \%)$
Good: $27(43.5 \%)$
Very Good: $21(33.9 \%)$
Excellent: 7 (11.3\%)

Satisfaction level $(n=206)$

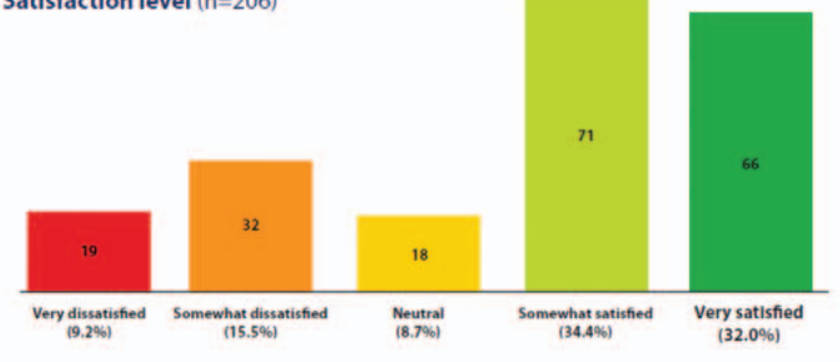

B

FIGURE 3. A and B, Baseline panel demographics and clinical characteristics. 


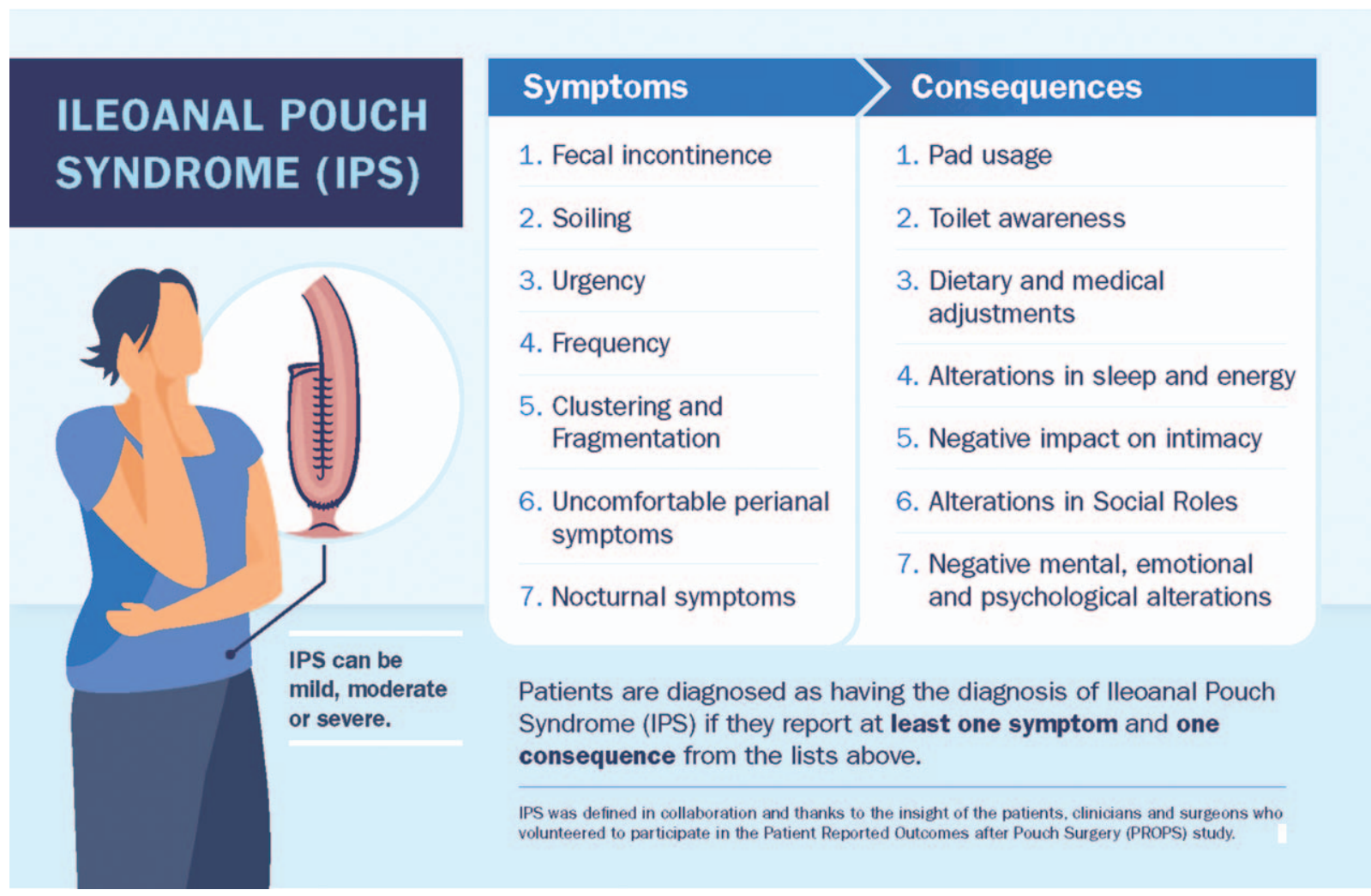

FIGURE 4. Final PROPS Delphi Study set of symptoms and accommodations. PROPS indicates patient reported outcomes after pouch surgery.

in the Core Outcome Measures in Effectiveness Trial. ${ }^{12}$ Patientcentered core outcome sets have been developed for several topics in colorectal surgery, such as perianal Crohn fistula disease ${ }^{13}$ and low anterior resection syndrome after rectal cancer surgery. ${ }^{14}$ Such outcome sets provide a useful starting point in the comprehension of experiences of patients treated for various conditions and allows research on how to improve symptoms that may be bothersome to patients. There has been no research defining the amalgamation of symptoms experienced by IPAA patients until the present consensus effort.

The patient reported outcomes after pouch surgery (PROPS) Delphi consensus study represents the first patient-centered effort in the surgical literature that has aimed to identify what functional outcomes patients consider important after pouch surgery, thus defining the concept of IPS - or the amalgamation of symptoms that are specific to having an ileoanal pouch. Our robust methodology included vital input from patients in study design, execution, participation, and analysis, balanced with opinions from surgeons, gastroenterologists, and specialist nurses. Ultimately, this iterative process identified 7 symptoms and 7 consequences of pouch function that were highpriority and should be at the core of any future study evaluating pouch function, with further research on those items that may lead to a deteriorated quality of life in pouch patients.

Our consensus process affirmed that many patients undergoing an ileal pouch experience an improved quality of life when compared to before surgery. In addition, our study confirmed that some of the symptoms that were the focus of previous studies, such as fecal incontinence, soiling, excessive number of bowel movements, and urgency, are important to patients. However, the study also highlighted several additional key symptoms that were not uniformly reported in previous studies including unpredictable bowel movements, altered patterns of bowel function (fragmentation, clustering, incomplete evacuation), nocturnal symptoms, and perianal pain. Finally - and most importantly - patients with pouches reported having to make multiple accommodations or changes in behavioral, dietary, and social/emotional aspects of their lives after pouch creation. These accommodations can be life-long and important in fully understanding the patient experience.

Prior studies have focused on an arbitrary set of functional outcomes deemed important by clinicians, typically surgeons, who study this population. On our previous review of the literature, we highlighted that parameters typically studied include fecal incontinence, 24-hour stool frequency, urgency, seepage, and the use of antidiarrheals. ${ }^{8}$ Furthermore, 3 clinical scoring systems have been described in the literature. The Oresland score ${ }^{15}$ was developed in 1989 in a unilateral fashion primarily by surgeons and has been utilized in a number of studies to analyze determinants of pouch dysfunction. The second score, the Pouch Function Score (PFS), ${ }^{16}$ was developed to assess patient reported pouch function, however, the starting list of symptoms used to derive the PFS was created by surgeons conducting the study and again includes 24-hour stool frequency, nocturnal stool frequency, incontinence, and the use of anti-diarrheal medications as the major domains. Lastly, the pouch dysfunction score ${ }^{17}$ was created with a cohort of 1757 patients that quantified function using a list generated by a combination of 6 expert surgeons and 10 randomly selected pouch patients. In our review of the literature, this score has not been widely used and the list of symptoms is not comprehensive. 
Nearly all of the prior studies evaluating outcomes and creating scores for pouch function are hampered by 2 important fundamental limitations: (1) heterogenous selection of primary outcome measurements; and (2) a paternalistic, clinician-driven approach to which specific symptoms are studied. This is a major gap in the current surgical literature, as we know that clinicians' perceptions of what symptoms are important to patients are often skewed and miscalculated, specifically in patients with pouches. ${ }^{9}$ Data from over 150 patients at the principal investigator's institution suggests that approximately $50 \%$ of patients experience varying degrees of fragmentation and incomplete evacuation, symptoms that are rarely evaluated in studies of pouch function. ${ }^{8}$ Taken together, it is clear that the outcomes used to define pouch function or dysfunction should not be determined unilaterally by surgeons, and the views of patients and other clinicians involved in the care of UC patients with pouches must be considered, as they were in the PROPS consensus study.

There are several limitations that should be discussed when considering the results of this study. First, recruitment of patients was done using an online social media strategy. Although this had the potential to recruit large numbers of patients that interact with the $\mathrm{CCF}$ social media page, it also introduces sampling bias to participants that are active in the online community and biased against older patients or those from poorer socio-economic backgrounds. This is in contrast to more traditional methods of recruiting patients known to surgeons at IBD centers, which also may be inherently biased. Despite this, we felt that our cohort of patients had a broad range of experiences and satisfaction levels with their pouch, and conversation was not dominated by overwhelmingly positive or negative viewpoints. Furthermore, although we attempted to include a comprehensive list of starting domains, and additional patient suggested items, it is possible that some important symptoms were not discussed. Therefore, the final set of symptoms/accommodations should be viewed as the bare minimum that should be studied, and additional symptoms can be added based on study characteristics. Lastly, we do not present clinical outcomes such as pouchitis or pouch failure - importantly, the aim of this study was not to quantify how much each symptom impacts quality of life associated with various clinical outcomes. The primary goal was to develop a comprehensive list of all of the symptoms that patients feel are most important.

Now that the core set of symptoms and accommodations experienced by patients with pouches has been identified in the PROPS study, the next hurdle is to develop a clinically useful scoring system that can quantify the range and severity of symptoms experienced by ileoanal pouch patients and their correlation with quality of life parameters. This score would theoretically help identify those patients whose pouch function symptoms may fall into a range where these symptoms have a severe negative impact on quality of life. The proposed score will help identify patients with IPS meeting a designated threshold score and also be able to assess the efficacy of medical and surgical interventions to improve the quality of life in the patients with IPS. This effort has received additional support from the Crohn's and Colitis Foundation and is currently ongoing.

\section{ACKNOWLEDGMENT}

The authors would like to acknowledge Lindsey Ruane and Cameron Hunt for their contributions to the study.

\section{REFERENCES}

1. Parks AG, Nicholls RJ. Proctocolectomy without ileostomy for ulcerative colitis. Br Med J. 1978;2:85-88.

2. Fazio VW, Ziv Y, Church JM, et al. Ileal pouch-anal anastomoses complications and function in 1005 patients. Ann Surg. 1995;222:120-127.

3. Fazio VW, O'Riordain MG, Lavery IC, et al. Long-term functional outcome and quality of life after stapled restorative proctocolectomy. Ann Surg. 1999;230:575-586.

4. McKenna NP, Dozois EJ, Pemberton JH, et al. Impact of sex on 30-day complications and long-term functional outcomes following ileal pouch-anal anastomosis for chronic ulcerative colitis. Int $J$ Colorectal Dis. 2018;33: 619-625.

5. De Buck van Overstraeten A, Wolthuis AM, Vermeire S, et al. Long-term functional outcome after ileal pouch anal anastomosis in 191 patients with ulcerative colitis. J Crohn's Colitis. 2014;8:1261-1266.

6. Juul T, Ahlberg M, Biondo S, et al. International validation of the low anterior resection syndrome score. Ann Surg. 2014;259:728-734.

7. Emmertsen KJ, Laurberg S. Low anterior resection syndrome score: development and validation of a symptom-based scoring system for bowel dysfunction after low anterior resection for rectal cancer. Ann Surg. 2012;255:922-928.

8. Lee GC, Cavallaro PM, Savitt LR, et al. Bowel function after J-pouch may be more complex than previously appreciated: a comprehensive analysis to highlight existing knowledge gaps. Dis Colon Rectum. 2020;63:207-216.

9. Brandsborg S, Chen TY, Nicholls RJ, et al. Difference between patients' and clinicians' perception of pouch dysfunction and its impact on quality of life following restorative proctocolectomy. Color Dis. 2015;17:0136-O140.

10. McNair AGK, Heywood N, Tiernan J, et al. A national patient and public colorectal research agenda: integration of consumer perspectives in bowel disease through early consultation. Color Dis. 2017;19:075-O85.

11. Harris PA, Taylor R, Thielke R, et al. Research electronic data capture (REDCap)-a metadata-driven methodology and workflow process for providing translational research informatics support. J Biomed Inform. 2009; 42:377-381.

12. Williamson P, Clarke M. The COMET (core outcome measures in effectiveness trials) initiative: its role in improving cochrane reviews. Cochrane Database Syst Rev. 2012;13:ED000041.

13. Sahnan K, Tozer PJ, Adegbola SO, et al. Developing a core outcome set for fistulising perianal Crohn's disease. Gut. 2019;68:226-238.

14. Keane C, Fearnhead NS, Bordeianou L, et al. International consensus definition of low anterior resection syndrome. Colorectal Dis. 2020;22: $331-341$.

15. Öresland T, Fasth S, Nordgren S, et al. The clinical and functional outcome after restorative proctocolectomy - a prospective study in 100 patients. Int J Colorectal Dis. 1989;4:50-56.

16. Lovegrove RE, Fazio VW, Remzi FH, et al. Development of a pouch functional score following restorative proctocolectomy. Br J Surg. 2010; 97:945-951.

17. Brandsborg S, Nicholls RJ, Mortensen LS, et al. Restorative proctocolectomy for ulcerative colitis: development and validation of a new scoring system for pouch dysfunction and quality of life. Color Dis. 2013;15:e719-e725. 